\title{
The use of parametric modeling and rapid prototyping in teaching graphic expression
}

\section{SIGRADI2018 TECHNOPOLITICAS xxii congresso da sociedade iberoamericana de gráfica digital 22th conference of the iberoamerican society \\ of digital graphics \\ 07|08|09|novembro|2018 iau usp | são carlos | sp br}

\author{
Leticia Teixeira Mendes \\ Universidade Federal de Pernambuco | Brazil | leticia.mendes@ufpe.br \\ Elton Cristovão da Silva Lima \\ Universidade Federal de Pernambuco | Brazil | eltoncristovao1995@hotmail.com \\ Cristiana Griz \\ Universidade Federal de Pernambuco | Brazil | crisgriz@gmail.com
}

\begin{abstract}
The introduction of new technologies in design areas is due, in large part, to the rapid development of the computer industry. This new paradigm has created tools and creative possibilities in the routine of several professionals. The Graphic Expression Department is responsible for disciplines that include the knowledge of Graphical Geometry and different approaches to graphically represent projects from different areas of knowledge. Thus, the present experiment presents a possibility of updating the teaching of representation from the introduction of Shape Grammar and through digital tools, in particular, the use of parametric design, visual programming and rapid prototyping.
\end{abstract}

Keywords: shape grammar; parametric design; graphic geometry; visual programming.

\section{INTRODUÇÃO}

A introdução de novas tecnologias nas áreas de projeto se deve, em grande parte, ao rápido desenvolvimento da indústria da computação. Esse novo paradigma tem criado ferramentas e possibilidades criativas na rotina de diversos profissionais como artistas, arquitetos, designers e engenheiros. Recentemente, a partir da inserção das ferramentas de prototipagem rápida $(\mathrm{PR})$ e fabricação digital (FD), tornou-se possível utilizar modelos geométricos digitais diretamente na produção de objetos e componentes em escala real.

Para acompanhar essas inovações no campo do projeto, a universidade vem, cada vez mais, propondo novas metodologias de ensino que incorporem tais tecnologias no processo didático. Na área do ensino da geometria gráfica essa mudança, que vem ocorrendo há algumas décadas (PUPO, 2008; PUPO et al., 2008), está sendo acelerada para que 0 jovem profissional não fique defasado em relação ao mercado. Segundo Oxman (2008, p. 99):

\footnotetext{
A busca por novas estruturas educacionais se deve aos impactos pedagogicamente únicos do projeto digital. Vários pesquisadores e educadores começaram a abordar a necessidade de integrar o projeto digital na educação de projeto arquitetônico, investigando várias formas de agenda pedagógica (Tradução nossa).
}

O estudo das técnicas de geométrica gráfica se enquadra na área de comunicação. Assim como a linguagem falada e escrita, a gráfica também possui uma "gramática" para poder ser bem entendida, tanto por quem escreve, quanto por quem lê. Enquanto nas duas primeiras, o meio para se expressar são as palavras, existentes nas mais variadas línguas, na expressão gráfica este meio tem sido, tradicionalmente, o desenho.

Entretanto, como comentado, as novas tecnologias têm substituído o tradicional desenho - mesmo aqueles feitos em "pranchetas digitais"1, por um tipo de "modelagem virtual" (ou real, no caso da PR e da FD) de formas geométricas. Vale ressaltar que, assim como o desenho, para a execução das modelagens continua sendo necessário, e indispensável, o conhecimento da geometria gráfica.

Isso porque, como é sabido, aqueles que detêm o conhecimento desses métodos da geometria gráfica apresentam maior liberdade criativa (Flório, 2011). Ou seja, quanto maior o grau de conhecimento das formas geométricas e das suas possibilidades de representação, maior a capacidade e liberdade criativas. Nesse sentido, a geometria gráfica pode ser considerada como a ciência fundamental para a pesquisa sobre a forma.

Pensando nisso, o Departamento de Expressão Gráfica da Universidade Federal de Pernambuco, responsável por disciplinas que contemplam saberes da Geometria Gráfica e diferentes abordagens para representar graficamente um projeto, vem reformulando o conteúdo e a metodologia de ensino de várias disciplinas. Uma delas é a de Desenho Geométrico, responsável pelo

\footnotetext{
${ }^{1}$ Desenhos feitos em softwares como o AutoCAD, por exemplo, executados com a mesma lógica do desenho desenvolvido com lápis e papel são, genericamente, chamados de "prancheta digital".
} 
conhecimento das propriedades gráficas de todas as formas geométricas bidimensionais e sua aplicação na arquitetura, urbanismo e paisagismo.

Objetivando a introdução não apenas do conhecimento de novas tecnologias de criação e representação de formas geométricas, mas também de uma nova metodologia projetual, foi introduzido o conceito de sistemas generativos de projeto para dar suporte ao ensino de polígonos (um dos itens programáticos da disciplina). Fischer e Herr (2001), definem um sistema generativo como uma metodologia sistemática para a produção de soluções projetuais, não só no que se refere a criar produtos, mas principalmente o processo de criação desses produtos.

Dessa maneira, o experimento apresentado no presente artigo, mostra uma possibilidade de atualização do ensino de geometria gráfica a partir da introdução de um sistema generativo de projeto - a Gramática da Forma (Stiny e Gips, 1971), e por meio das ferramentas digitais, em especial, o uso de modelagem paramétrica (Monedero, 2000) e prototipagem rápida. Assim, pretende-se contribuir para a introdução de novos métodos de projeto no ensino, bem como no desenvolvimento de novas ferramentas e abordagens projetuais introduzidos na formação profissional.

\section{GRAMÁTICA DA FORMA E PROJETO PARAMÉTRICO COMO EXPERIÊNCIA DIDÁTICA}

O Movimento do Métodos de Projeto constituiu um período de conferências e publicações sobre métodos de projeto nas áreas de arquitetura, engenharia e desenho industrial na década de 1960, influenciando, de maneira significativa, o ensino e a prática dessas áreas de conhecimento (Mendes, 2014). A partir dessas discussões, duas abordagens vêm sendo empregadas no sentido de otimizar o processo projetual e geração de alternativas: a parametrização (parametric design) e o projeto baseado em regras (rule-based design). A primeira estratégia consiste em definir relações topológicas, sendo a definição das medidas precisas uma fase subsequente do projeto. Em geral essas dimensões podem ser selecionadas a partir de um intervalo desejável, com valores mínimos e máximos, resultando em maior variedade de soluções (Monedero, 2000).

Como comentado, o presente trabalho tem como objetivo apresentar o experimento realizado em uma disciplina de Geometria Gráfica para o curso de Arquitetura e Urbanismo, a partir do uso do projeto paramétrico e do formalismo da Gramática da Forma. Isso porque entendese que a introdução dessas abordagens pode contribuir para uma compreensão mais interativa dos elementos geométricos e suas propriedades.

Segundo Flório (2011), os antigos conhecimentos de geometria são fundamentais para compreender e projetar formas de grande complexidade. $\mathrm{O}$ autor propõe que se "faça uma re-valorização destes conhecimentos diante das facilidades trazidas pelas novas tecnologias de representação e de simulação de espaços" (Flório, 2011, p.44).

Nesse contexto, existem ferramentas ainda pouco exploradas no ensino de geometria, constituídas pelos ambientes de modelagem paramétrica. A partir do uso destes instrumentos, como por exemplo o Grasshopper, é possível que o usuário desenvolva modelos por meio de um processo indireto de geração de formas, sendo necessários conhecimentos de programação computacional, matemática e lógica para explorar essas ferramentas no processo projetual (Pereira; Vaz, 2013).

Oxman (2006), define que o modelo de design associativo de formação se baseia em técnicas de modelagem paramétricas, possibilitando explorar a geometria associativa, cujas relações entre os objetos paramétricos são explicitamente descritas, estabelecendo interdependências entre os vários objetos. Diferentes valores atribuídos podem gerar múltiplas variações enquanto se mantém as condições topológicas. Dessa maneira, pode-se dizer que a modelagem paramétrica tem a necessidade de um ambiente computacional e faz uso de algoritmos ${ }^{2}$ para estabelecer definições, além de buscar a otimização de soluções.

Paralelamente, desde os anos 1960, diversas abordagens estruturalistas ${ }^{3}$ vêm sendo aplicadas no processo de projeto em arquitetura. Uma delas é o projeto baseado em regras - rule-based design (Broadbent, 1970), que consiste em definir situações em que determinado elemento pode ser conectado a outro, sendo pre-definida a maneira que isso pode ocorrer. Este método permite grande variedade de resultados, uma vez que a ordem da aplicação das regras pode originar composições completamente diferentes. Dentro desse contexto, o formalismo denominado Gramática da Forma possibilita a combinação dessas duas abordagens projetuais: a parametrização e o uso de regras compositivas resultando em um número ainda maior de possibilidades e geração de soluções (Mendes, 2018).

O sistema algorítmico definido como Gramática da Forma foi criado na década de 1970 por George Stiny e James Gips e consiste em um sistema de geração de formas baseado em regras, desenvolvido a partir das teorias da gramática generativa do linguista Noam Chomsky e do sistema de produção do matemático Emil Post (Celani et al., 2006). Ao longo dos anos, a Gramática da Forma vem sendo aplicada para resolução de grande variedade de problemas projetuais, investigando como gerar soluções a partir de uma forma inicial, por meio de sucessivas aplicações de regras de forma (Duarte, 2007; Knight, 2000; Celani et al., 2006). Desde então, diversos tipos de gramáticas foram elaboradas e aplicadas na arquitetura para a geração sistemática de arranjos espaciais, como por exemplo, aplicado à problemática de habitação de interesse social (Duarte, 2007; Mussi, 2011; Mendes, 2014) e na área de planejamento urbano (Beirão, 2012; Duarte et al., 2006 e Paio e Turkienicz, 2010).

\footnotetext{
2 De maneira simplificada, podemos fazer a analogia de um algoritmo com uma receita que apresenta, passo a passo, os procedimentos necessários para a resolução de uma tarefa. A introdução de algoritmos de parametrização, implementados em ferramentas computacionais no processo projetual, permite a exploração de grandes quantidades de alternativas de forma dinâmica e rápida, aumentando as possibilidades de investigação de diferentes variáveis.

3 Segundo Colin (2009), o estruturalismo é uma abordagem das ciências humanas que objetiva analisar um campo específico como um sistema complexo de partes inter-relacionadas.
} 
As gramáticas da forma podem ser analíticas ou originais (Duarte, 2007). Sendo analíticas quando usadas como ferramenta de análise de um grupo de projetos - o corpus, descrevendo-os através de uma única linguagem que os represente. Consideram-se originais quando é concebida para criar novos projetos, que atendam a requisitos pré-estabelecidos.

Podemos incluir ainda um terceiro tipo, a gramática paramétrica, uma vez que as primeiras definições de gramática da forma já sugeriam seu potencial de gerar grande variedade de resultados, quando certos valores (parâmetros) são deixados em aberto para serem definidos em uma fase subsequente (Celani et al., 2006). A partir do desenvolvimento das tecnologias computacionais tornou-se possível - e recorrente, em diversas áreas de conhecimento (design, artes e arquitetura) a implementação de Gramáticas da Forma em ambiente de modelagem paramétrica visual, como veremos no experimento descrito neste artigo.

As regras de uma Gramática da Forma definem transformações geométricas que serão aplicadas nas formas que compõe o vocabulário da gramática, como: rotação, translação e reflexão. Uma gramática pode ser considerada um sistema generativo paramétrico, a partir do momento que a aplicação de dada regra afeta um conjunto de sub-regras; e com um número finito de entrada de dados, pode-se gerar uma quantidade indefinida de soluções (Santos, 2009).

\section{METODOLOGIA}

A metodologia proposta para o exercício projetual se baseia no método da pesquisa-ação, proposto por Kurt Lewin, nos anos 1940 e na prática reflexiva, definida por Schön, em 1983 (Lewin, 1946; Schön, 1983). O método desenvolvido por Lewin é principalmente uma estratégia para o desenvolvimento de professores e pesquisadores que se utilizam de suas pesquisas para aprimorar sua didática, e como consequência, o aprendizado de seus alunos.

O livro "Educando o profissional reflexivo: um novo design para o ensino e a aprendizagem" (Schön, 2000), discute a experimentação nos ateliês de projetos arquitetônicos como um modelo educacional para a reflexão-na-ação. Assim, o autor argumenta que não há uma ciência geral que ensine a prática projetual, a não ser nos ateliês de projeto, sendo um exemplo típico de ensino prático reflexivo. Ainda sobre o processo de prática projetual em ateliês de arquitetura, o autor sugere que:

Implicam um tipo de aprender fazendo, em que os alunos começam a praticar, juntamente com os que estão em idêntica situação, mesmo antes de compreenderem racionalmente o que estão a fazer. Nos ateliês de projeto arquitetônico, por exemplo, os alunos começam por desenhar antes de saberem o que é o projeto. Nos primeiros tempos toda a gente se queixa da confusão (Schön, 1997).

Assim, a atividade apresentada neste artigo - um workshop de modelagem paramétrica para desenvolvimento de cobogós $^{4}$ - exemplifica a aplicação

${ }^{4} \mathrm{O}$ cobogó é um sistema vazado usado como elemento de vedação e proteção com o intuito de permitir a ventilação e filtrar a incidência solar, constitui uma estratégia passiva de da metodologia, na qual está sendo proposta a atualização do conteúdo de desenho geométrico por meio de um experimento acerca da utilização da parametrização e da prototipagem rápida no ensino da graduação em arquitetura e urbanismo.

Como comentado, o formalismo da Gramática da Forma já foi aplicado em diferentes áreas de conhecimento. Assim, utilizou-se esse método para analisar e gerar desde objetos do cotidiano, como cadeiras (Knight, 1980; Barros et al., 2011) ou cerâmicas (Costa e Duarte, 2015) à habitações (Duarte, 2007) e planos urbanísticos (Beirão, 2012; Duarte e Beirão, 2012). Neste experimento optou-se pela escolha do elemento arquitetônico 'cobogó' como objeto de estudo. Essa escolha foi feita pois este objeto é concebido através de uma composição formal, que permite a introdução do conteúdo da disciplina (polígonos e suas propriedades formais), excluindo a preocupação funcional, como por exemplo: a função de sentar de uma cadeira ou a legislação (e outras variáveis) que precisam ser consideradas para criação de um plano urbano. Dessa maneira, a proposta do exercício exige que os alunos se concentrem apenas na composição estética e formal do cobogó, explorando principalmente, a relação entre as formas bidimensionais para sua composição.

Dentro desse contexto, utilizou-se o ambiente de modelagem paramétrica visual Grasshopper associado ao programa de modelagem 3D Rhinoceros. Essa ferramenta para a implementação de definições de algoritmos generativos é amplamente utilizada em pesquisas que se embasam no uso de gramáticas da forma e modelagem paramétrica em workshops com estudantes de graduação (Beirão et al., 2015; Vaz, 2011)

Essa linguagem de programação visual possibilita a construção de geometrias através da conexão de componentes e parâmetros (Khabazi, 2010). Por meio desses elementos, é permitida a entrada e saída de dados (Input e Output) que executam funções predefinidas, podendo criar, alterar e desconstruir formas, além de possibilitar o encontro de diversas soluções projetuais. De acordo com a figura 1, o componente do Grasshopper Extrude (que permite fazer a extrusão de uma forma) possui os dados de entrada (input) da forma que receberá a ação de extrusão - nesse exemplo, um círculo - e a altura (vetor Z) controlada por uma barra de rolagem (slider) que permite variar esse parâmetro (altura).

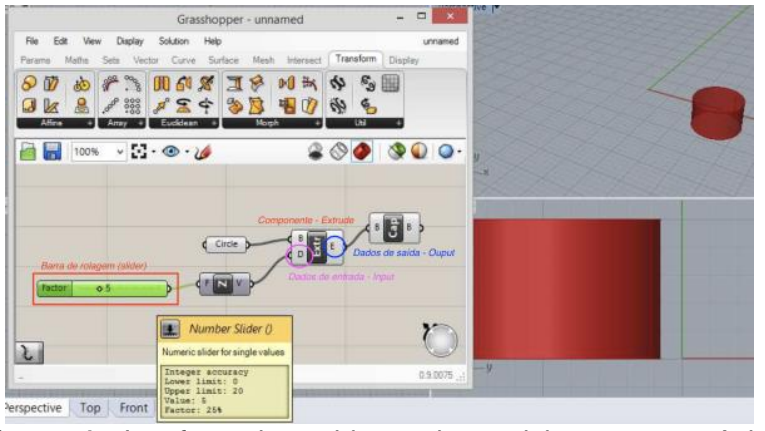

Figura 1: Interface do ambiente de modelagem paramétrica visual Grasshopper e exemplo de componente, slider, dados de entrada e de saída. Fonte: Autor, 2018.

amenização climática utilizada na arquitetura moderna brasileira (Santana Neto e Silva, 2016). 
Considerando que o experimento foi fundamentado no uso do ambiente paramétrico e de um sistema generativo, torna-se necessário a definição dos elementos que compõem uma Gramática. A primeira etapa para se desenvolver uma Gramática da forma é apresentar um conjunto de formas definido como vocabulário de formas. Após essa etapa, são definidas as relações espaciais entre as formas e, posteriormente, são definidas as regras de transformação do tipo $A \rightarrow B$, que podem ser aditivas ou subtrativas, por exemplo. Ou seja, a regra é composta por uma forma do lado esquerdo (a forma que deverá ser identificada) e uma relação espacial do lado direito da regra (que deverá substituir a forma da direita). Entre os dois elementos o símbolo "seta" definirá a relação: ao encontrar $A$, substitua por $B$ (Mendes, 2014). Com as regras definidas, pode-se aplica-las sistematicamente, gerando as derivações (Figura 02).

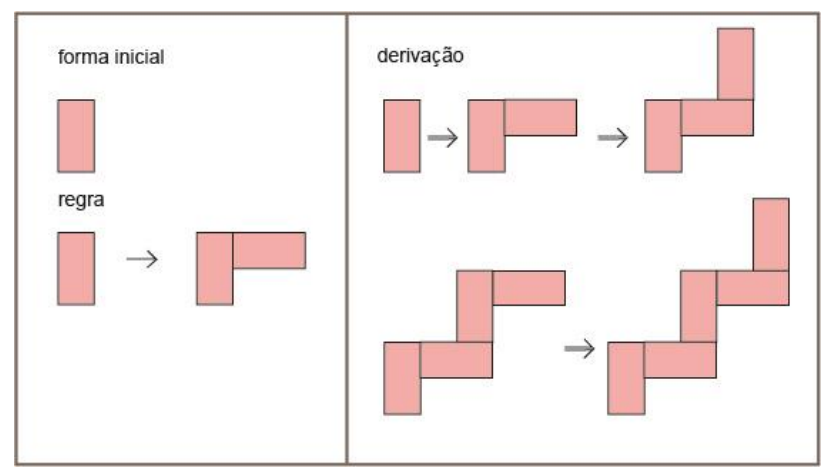

Figura 2: Exemplo de gramática da forma - forma inicial, regra e derivação. Fonte: Mendes, 2018.

Na presente pesquisa, foi desenvolvida uma gramática da forma paramétrica para geração de cobogós. O vocabulário definido foram elementos geométricos básicos como o círculo, quadrilátero e triângulo, uma vez que a ementa da disciplina de Geometria Gráfica 2D contempla a representação de figuras geométricas planas e sua construção.

\section{O EXPERIMENTO: UMA GRAMÁTICA DA FORMA PARA GERAÇÃO DE COBOGÓS}

A disciplina Geometria Gráfica 2D é responsável pelo aprendizado das propriedades das formas geométricas bidimensionais. $\mathrm{O}$ experimento foi feito, a princípio, para o tema 'polígonos', cujo foco é que o aluno aprenda suas propriedades formais e seus elementos constitutivos conhecimentos que vão permitir que o aluno construa diversos tipos de polígonos, seja no desenho tradicional, seja através de softwares de modelagem paramétrica.

O workshop ministrado realizou-se com a participação de 42 alunos divididos em duplas ou grupo de três estudantes. Num primeiro momento, eles foram instruídos para criar três elementos geométricos básicos - círculo, triângulo e quadrilátero no ambiente de modelagem paramétrica visual Grasshopper. Essa atividade requereu conhecimento prévio de conceitos de desenho geométrico como por exemplo: circuncentro, ortocentro, incentro, raio, diâmetro, além dos vários tipos de triângulos e polígonos com quatro ou mais lados (Figura 3).

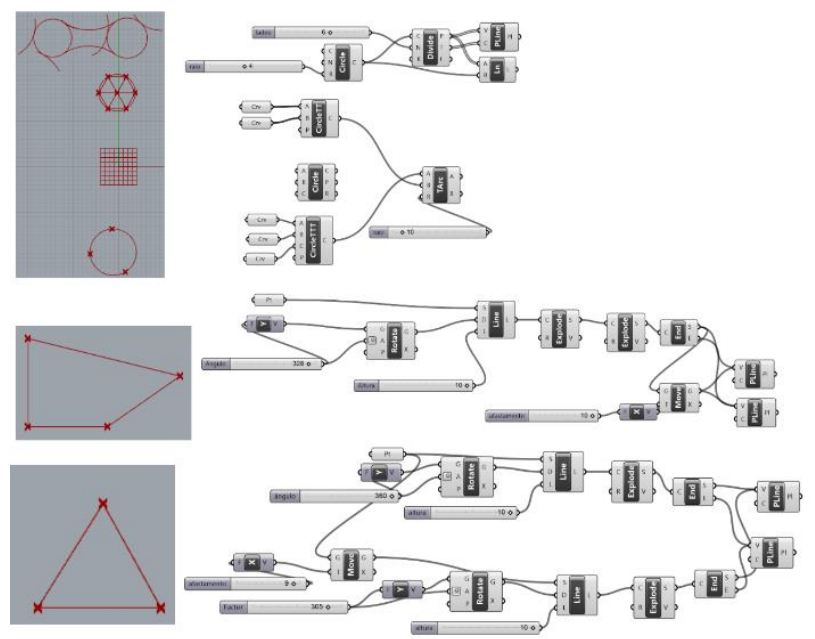

Figura 3: Exemplos de implementação no ambiente de modelagem paramétrica visual Grasshopper para construção de formas geométricas e construção de triângulos e suas propriedades: circuncentro, ortocentro, incentro (à direita). Fonte: Autor, 2018.

Após a familiarização com a interface do Grasshopper, feita através da criação de elementos básicos, a gramática da forma implementada foi testada pelos alunos. Para tanto, os alunos manipularam as barras de rolagem (sliders) nos códigos (Figura 4). As regras implementadas no ambiente de modelagem paramétrica permitiam alterar a quantidade de lados do polígono e aplicar transformações geométricas como: rotação, e translação. Com isso foi possível criar diversos modelos de cobogós, usando polígonos como forma inicial e modificando suas formas e relações espaciais.

\section{VOCABULÂRIO DE FORMAS}
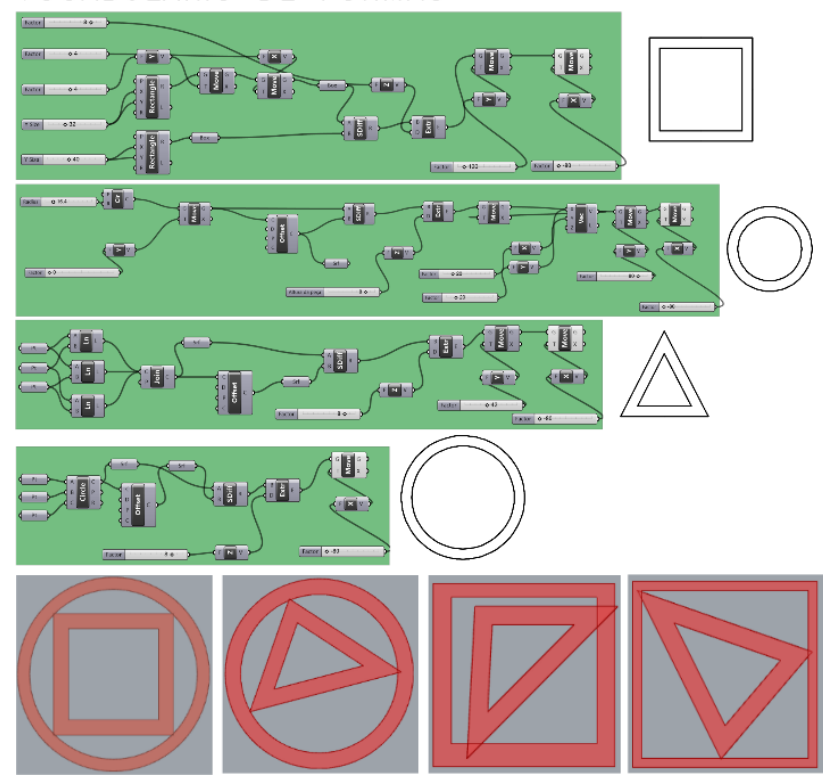

Figura 4: Implementação no ambiente de modelagem paramétrica visual Grasshopper com o vocabulário de formas da gramática de cobogós (A) e exemplos de cobogós gerados pelos alunos a partir da implementação (B). Fonte: Autor, 2018.

Como parte dos resultados do experimento, os cobogós criados a partir da gramática implementada, foram produzidos em impressora 3D. Para isso, utilizou-se uma tecnologia aditiva de prototipagem rápida - Modelagem por Deposição Fundida (em inglês Fused Deposition 
Modeling - FDM) - para produção dos modelos físicos. Segundo Pallasma (2011), a materialização da forma contribui no desenvolvimento do pensamento projetual e possibilita a comunicação, através do tato, tanto quanto a visão (Figura 5).

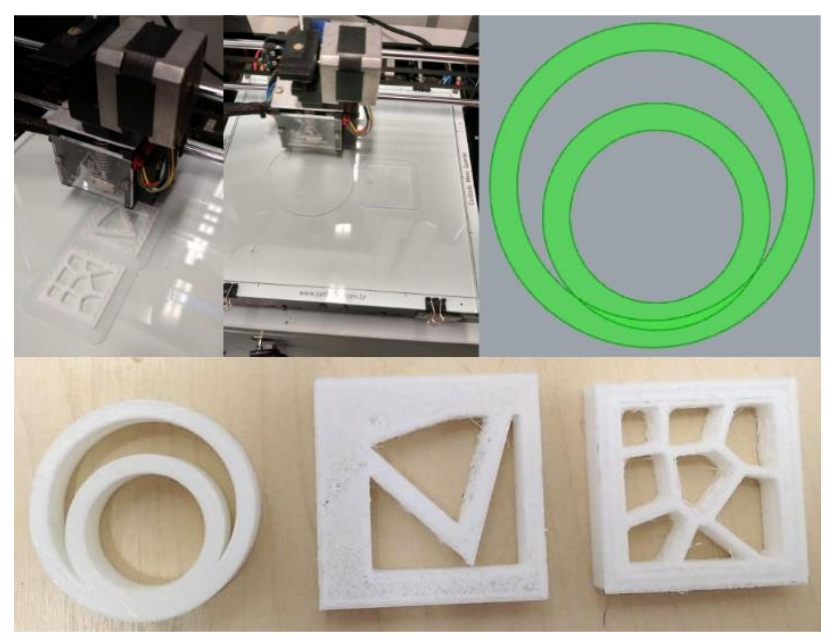

Figura 5: Processo de impressão 3D; Exemplo de um cobogó desenvolvido pelos alunos e exemplos de cobogós produzidos em impressora 3D. Fonte: Autor, 2018.

Dessa maneira, o processo de prototipagem permitiu materializar diferentes cobogós produzidos por meio da mesma gramática da forma, sendo assim, estes pertecem à uma mesma "linguagem" - apresentando as mesmas características, no entanto, com variações (Figura 6).

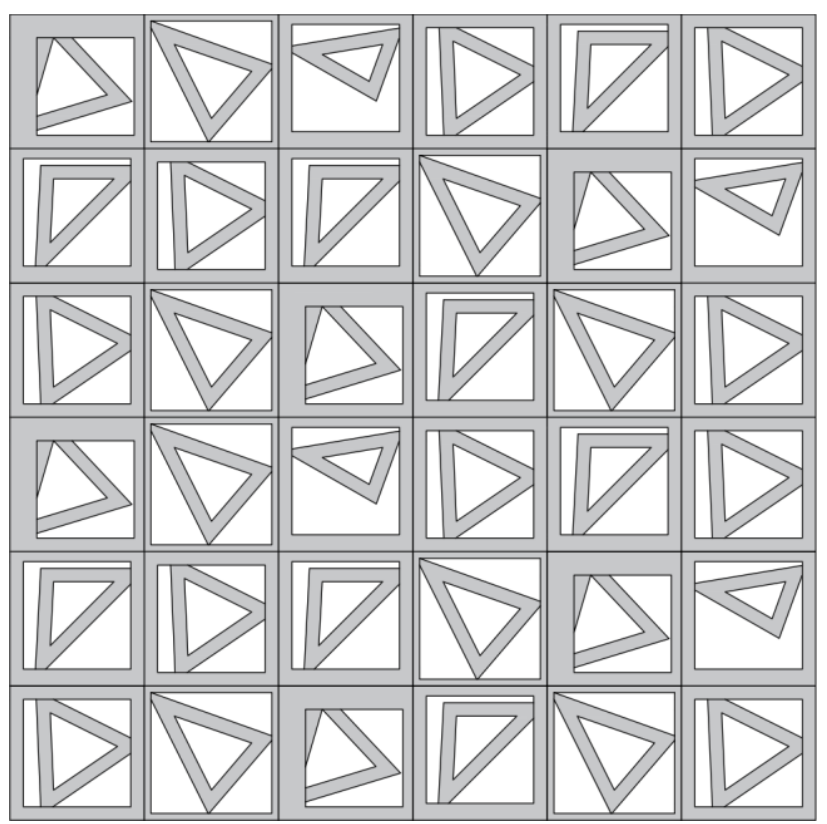

Figura 6: Exemplo de composição de um painel com diferentes cobogós gerados a partir da gramática da forma implementada em ambiente de modelagem paramétrica. Fonte: Autor, 2018.

A associação de um sistema generativo de projeto - a Gramática da Forma - ao potencial da prototipagem rápida, suscitou questionamentos contrapondo os modelos de produção em série (mass production) e o conceito de personalização em massa (mass customization) definido por autores como Stan Davis (1996), Tseng e Jiao (2001), Pine (1993), e Kaplan e Haenlein (2006). Dentro desse paradigma, a Gramática da Forma permite criar grandes quantidades de alternativas (ou diversos cobogós) de forma dinâmica e automatizada, ao passo que a tecnologia de prototipagem rápida possibilita sua materialização individualizada, sem gerar custos extras.

\section{RESULTADOS}

O experimento mostrou que a modelagem paramétrica, ainda que se utilize das tecnologias computacionais, requer conhecimentos de geometria para explorar as potencialidades da ferramenta de maneira consciente e eficiente, contribuindo para uma dinâmica de transitar entre procedimentos dedutivos referentes às construções gráficas e indutivos referentes às associações de parâmetros (Terzidis, 2006). Dessa forma, pode-se considerar que as aulas prévias sobre os conceitos de geometria abordados continuam sendo muito relevantes para a construção do raciocínio quando os alunos partiram para o ambiente paramétrico, sendo esta segunda etapa também uma oportunidade dos alunos fixarem o conteúdo de polígonos.

Dos 42 alunos participantes desta atividade, apenas três já tinham trabalhado com programação. No entanto, a linguagem utilizada era o Java, e não a de programação visual. Por causa dessa experiência anterior, esses alunos consideraram a atividade e a interação com o ambiente de modelagem paramétrica visual Grasshopper relativamente fáceis, diferente do nível de dificuldade relatado pela maioria dos alunos participantes (Figura 7).
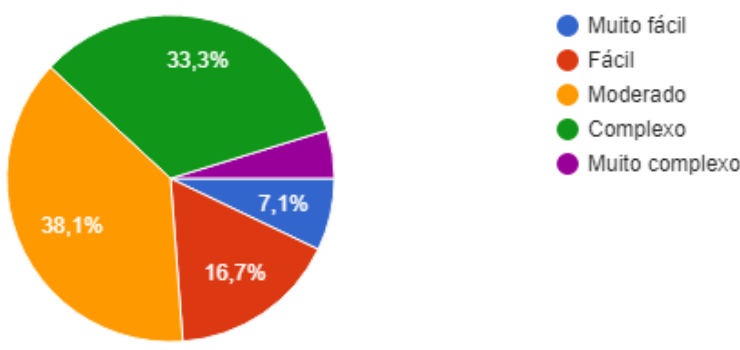

5

Figura 7: Gráfico de nível de dificuldade de interação com o Grasshopper. Muito fácil (3 alunos), fácil (7 alunos), moderado (16 alunos), complexo (14 alunos) e muito complexo (2 alunos).

De acordo com as respostas dos participantes, 61,9\% dos alunos (26) consideraram a interação com o Grasshopper 'muito fácil', 'fácil' ou 'moderada'. Esse dado pode ser explicado pelo fato de utilizarmos um ambiente de modelagem paramétrica visual, no qual os estudantes não precisaram ter conhecimento de programação com códigos escritos, mas podiam criar e manipular as definições por meio de componentes visuais, como exemplificado na figura 1. Além disso, os ícones podem ser encontrados de forma intuitiva e sua visualização ser passível de ajustes, permitindo alterar entre o desenho gráfico representativo ou o seu próprio nome.

Em relação às atividades propostas - criação de elementos geométricos paramétricos e manipulação da gramática da forma - os alunos acharam mais fácil criar o código da programação do que, apenas manipular as definições já prontas. Esse resultado demonstra que a implementação da programação desenvolvida pelos próprios estudantes do início ao fim, permite que eles compreendam a lógica subjacente sendo construída por meio dos componentes do Grasshopper. Ao passo que, 
fornecer uma definição pronta para se chegar a determinado resultado - como os cobogós - dificultou o entendimento do processo como um todo, fazendo com que os participantes apenas manipulassem os parâmetros, muitas vezes, de maneira inconsciente e automática.

No que tange o conhecimento da Gramática da Forma, percebe-se que, normalmente, nas aulas expositivas e no primeiro contato da turma com este assunto, os alunos têm dificuldade para compreender como funciona a lógica desse sistema generativo. No entanto, quando a gramática foi abordada de forma paramétrica, 34 dos 42 alunos afirmaram ter compreendido melhor.

De modo geral, 90,5\% (38) dos alunos disseram ter compreendido as potencialidades da abordagem projetual do projeto paramétrico. Entretanto, menos da metade da turma afirmou que usaria esta metodologia para projetar. Uma das possíveis razões da desmotivação para utilizar novamente essa abordagem em outras oportunidades, seja a complexidade do ambiente de modelagem paramétrica visual e a falta de incentivo para utilização de diferentes abordagens projetais no curso de Arquitetura e Urbanismo.

Um dado interessante foi que os próprios alunos afirmaram que é de suma importância ter conhecimento das abordagens projetuais (parametrização e projeto baseado em regras) apresentadas no workshop. Além disso, afirmaram que o entendimento prévio do conteúdo da disciplina, ou seja, de desenho geométrico, facilitaram a compreensão da lógica da implementação paramétrica. E 92,9\% (39) dos alunos afirmaram que o conhecimento de geometria (construção de polígonos, suas propriedades formais e seus elementos constitutivos) foi fundamental para possibilitar maior fluidez na assimilação do funcionamento do ambiente de modelagem paramétrica utilizado.

\section{DISCUSSÃO}

O presente artigo teve como objetivo apresentar o experimento realizado em uma disciplina de Geometria Gráfica para o curso de Arquitetura e Urbanismo, a partir do uso do projeto paramétrico, do formalismo da Gramática da Forma e do potencial da utilização de uma tecnologia de prototipagem rápida para materialização dos resultados.

As inquietações que nos levaram a este experimento foram construídas a partir da crítica ao ensino tradicional das disciplinas que contemplam saberes da Geometria Gráfica, partindo do pressuposto que esse conhecimento é fundamental para compreender e projetar formas de grande complexidade utilizando as tecnologias digitais contemporâneas.

Dentre as considerações já apresentadas neste trabalho, enfatizamos a importância de tal experimento na busca para atualizar o conteúdo ministrado em disciplinas de geometria, principalmente no que se relaciona à introdução de diferentes abordagens projetuais - como a parametrização e o projeto baseado em regras - no ensino e na formação de futuros profissionais. Essa experiência demonstrou a urgência de propormos novas metodologias de ensino que incorporem as tecnologias digitais na área de ensino da geometria gráfica, constituindo uma associação do conhecimento tradicional para a apropriação consciente dos recursos tecnológicos atuais.

Por meio das respostas dos participantes do experimento foi possível observar a importância de disponibilizar para os alunos ferramentas que lhes permitam, de uma maneira sistemática e objetiva (possibilitado pelo uso da Gramática da Forma e da modelagem paramétrica), explorar grandes quantidades de alternativas de forma dinâmica e automatizada. Assim, no intuito de contribuir na introdução de novos métodos projetuais no ensino de cursos de Arquitetura e Urbanismo, pretende-se desdobrar a metodologia aplicada neste experimento, para o conteúdo de uma disciplina de Geometria Gráfica, permitindo que os alunos tenham um período maior para assimilar o conhecimento e as ferramentas digitais e aplica-lo em problemas projetuais mais complexos.

\section{AGRADECIMENTOS}

O presente trabalho foi realizado com o apoio da FACEPE - Fundação de Amparo à Ciência e Tecnologia de Pernambuco (Processo APQ no 0781-6.04/15) e da PROPESQ/UFPE (Programa de Bolsa de Iniciação Científica UFPE/CNPq).

\section{REFERÊNCIAS}

BARROS, M., DUARTE, J., CHAPARRO, B. (2011). Thonet Chair Design Grammar : A Step towards the Mass Customization of Furniture. In: CAAD Futures 2011 : Designing Together. Liège, pp. 181-200.

BEIRÃO,J.N. (2012). CltyMaker. Designing Grammars for Urban Design. Architecture and the Built Environment, n. 5.

BEIRÃO, J. N.; MENDES, L. T.; CELANI, G. (2015). O uso do CIM (City Information Modeling) para geração de implantação em conjuntos de habitação de interesse social: uma experiência de ensino. Gestão e Tecnologia de Projetos, São Paulo, v. 10, n. 2, p. 101-112.

COSTA E.C., DUARTE J.P. (2015) Generic Shape Grammars for Mass Customization of Ceramic Tableware. In: Gero J., Hanna S. (eds) Design Computing and Cognition '14. Springer, Cham.

CELANI, G.; CYPRIANO, D.; GODOY, G.; VAZ, C. E. (2006). A gramática da forma como metodologia de análise e síntese em arquitetura. In: Conexão- comunicação e cultura. Vol. 5, p. 180-197. Caxias do Sul.

COLIN, S. (2009). As estruturas. Revista AU. Editora PINI: no 181. Abril, p.81.

DUARTE, J. P. (2007). Personalizar a habitação em série: Uma Gramática Discursiva para as Casas da Malagueira do Siza'. Lisboa: Ed. Fundação Calouste Gulbenkian.

DUARTE, J. P.; BEIRÃO, J. N. (2012). Towards a methodology for flexible urban design: designing with urban patterns and shape grammars. Environment and Planning B: planning and design, v. 38, n. 5, p. 879-902.

FISHER, T; HERR, C. M. (2001). Teaching generative design, In: Proceedings of the 4th International Generative Art Conference. Milão: Ed. SODDU.

FLÓRIO, W. (2011). Modelagem Paramétrica, Criatividade e Projeto: duas experiências com estudantes de arquitetura. In: Gestão e Tecnologia de Projetos. Vol. 6, N. 2, São Carlos, p. 43-66.

KHABAZI, Z. (2010). Generative Algorithms using Grasshopper. Morphogenesism education.

KNIGHT, T. (2000). Shape Grammars in education and practice: history and prospects, proceedings of International Journal of Design Computing, v. 2. Disponível em: 
<http://www.mit.edu/ tknight//JDC/ >. Acesso em: 23 jul. 2012

KNIGHT, T. (1980). The generation of Hepplewhite-style chaiback designs. Environment and Planning B : Planning and Design 7 : 227-238.

LEWIN, K. (1946). Action research and minority problems. Journal of Social Issues, n. 2, p. 34-36.

MENDES, L. T. (2014). Personalização de habitação de interesse social no Brasil: o caso da implantação urbana em conjuntos habitacionais. Tese de Doutorado. Universidade Estadual de Campinas.

MENDES, L. T. (2018). Gramática da forma: aplicações em habitação social. In: CELANI, G., SEDREZ, M. (Org.) Arquitetura Contemporânea e Automação: Prática e reflexão. ProBooks: São Paulo, pp. 41-54.

MONEDERO, J. (2000). Parametric design - a review and some experiences. Automation in Construction, 9 (4), 369-377.

MUSSI,

OXMAN, R. (2006). Theory and Design in the first digital age. In: Design Studies 27. Londres: Elsevier.

OXMAN, Rivka. (2008). Digital architecture as a challenge for design pedagogy: theory, knowledge, models and medium. Design Studies, Vol. 29. pp. 99-120.

PAIO, A.; TURKIENICZ, B. A Grammar for Portuguese Historical Urban Design. FUTURE CITIES [28th eCAADe Conference Proceedings / ISBN 978-0-9541183-9-6] ETH Zurich (Switzerland) 15-18 September 2010, pp.349-358

PALLASMA, J. (2011). As Mãos Inteligentes: A sabedoria Existencial e Corporalizada na Arquitetura. Porto Alegre: Bookman.

PEREIRA, N. S., VAZ, C. V. (2013). Parametrismo e Ensino de Geometria - As Superfícies de Felix Candela. Disponível em: $<$ http://wright.ava.ufsc.br/ grupohipermidia/graphica2013/tra balhos/PARAMETRISMO\%20E\%20ENSINO\%20DE\%20GE OMETRIA\%20AS\%20SUPERFICIES\%20DE\%20FELIX\%20 CANDELA.pdf.>

PUPO, R. T. (2008). Ensino da prototipagem rápida e fabricação digital para arquitetura e construção no Brasil: definições e estado da arte. PARC, v. 1, p. 1-19. Disponível em: <http://www.fec.unicamp.br/ parc> Acessado em Jun/2018.

PUPO, R., DUARTE, J. e CELANI, G. (2008). Introducing digital fabrication into the architectural curriculum: two similar experiences in different contexts. Congresso ECAADE. Antwerpen

SANTANA NETO, E. J., SILVA, R. C. (2016). Computação material: um estudo sobre a atualização geométrica de elementos vazados na arquitetura. In: SIGraDi - XX Congreso de la Sociedad Ibero-americana de Gráfica Digital. Buenos Aires. Disponível em: http://pdf.blucher.com.br.s3sa-east-

1.amazonaws.com/designproceedings/sigradi2016/479.pdf

SANTOS, L. F. B. S. (2009). Sistemas Generativos de Projecto: Integração de Ferramentas Digitais no Projecto de Arquitectura. Dissertação (Mestrado em Arquitetura) Instituto Superior Técnico, Universidade Técnica de Lisboa. $155 f$.

SCHÖN, D. (1983). The reflective practitioner: how professionals think in action. Nova York: Basic Books, 1983.

SCHÖN, D. (1997). FORMAR PROFESSORES COMO PROFISSIONAIS REFLEXIVOS. In: NÓVOA, A. (Coord.). Os Professores e a sua Formação. 3a ed. Lisboa, Publicações Dom Quixote.

SCHÖN, D. (2000). Educando o Profissional Reflexivo: um novo design para o ensino e a aprendizagem. Porto Alegre: Ed. Artmed.
STINY, G.; GIPS, J. (1971). Shape Grammars and the Generative Specification of Painting and Sculpture, in C V Freiman (ed) Proceedings of IFIP Congress 71 (Amsterdam: North-Holland) 1460- 1465. Republished in O R Petrocelli (ed), The Best Computer Papers of 1971 (Philadelphia: Auerbach) pp125-13.

TERZIDIS, K. (2006). Algorithmic Architecture. Burlington: Elsevier.

VAZ, C. E. V. (2011). Um método de ensino de projeto baseado em precedentes - sistemas generativos e ontolologias aplicados no ensino de arquitetura paisagística. Tese de doutorado. Universidade Estadual de Campinas. Campinas. 